PROCEEDINGS OF THE

AMERICAN MATHEMATICAL SOCIETY

Volume 131, Number 8, Pages 2491-2500

S 0002-9939(03)07046-1

Article electronically published on March 11, 2003

\title{
LIMITATIONS ON THE EXTENDIBILITY OF THE RADON-NIKODYM THEOREM
}

\author{
GERD ZEIBIG
}

(Communicated by N. Tomczak-Jaegermann)

\begin{abstract}
Given two locally compact spaces $X, Y$ and a continuous map $r: Y \rightarrow X$ the Banach lattice $\mathcal{C}_{0}(Y)$ is naturally a $\mathcal{C}_{0}(X)$-module. Following the Bourbaki approach to integration we define generalized measures as $\mathcal{C}_{0}(X)$ linear functionals $\mu: \mathcal{C}_{0}(Y) \rightarrow \mathcal{C}_{0}(X)$. The construction of an $L^{1}(\mu)$-space and the concepts of absolute continuity and density still make sense. However we exhibit a counter-example to the natural generalization of the Radon-Nikodym Theorem in this context.
\end{abstract}

\section{Notation AND BASIC DEFINITIONS}

Throughout the paper $X$ and $Y$ will denote locally compact spaces and $r: Y \rightarrow$ $X$ will be a fixed continuous function.

We use standard notation as in 3, 5]. In particular $\mathcal{C}_{0}(X)$ stands for the space of all complex-valued continuous functions on $X$ which vanish at infinity and $\mathcal{C}_{b}(X)$ is the space of all complex-valued bounded continuous functions on $X$. The injective and the projective tensor products are respectively denoted $\ddot{\otimes}$ and $\hat{\otimes}$. The most relevant feature of the injective tensor product for the present work is that we can identify $\mathcal{C}_{0}(X \times Y)$ with $\mathcal{C}_{0}(X) \dot{\otimes} \mathcal{C}_{0}(Y)[2]$.

Recall that a Banach module $M$ over the Banach algebra $\mathcal{C}_{0}(X)$ (under the pointwise operations) is a Banach space together with a contractive bilinear actionmap $\mathcal{C}_{0}(X) \times M \dot{\rightarrow}$. If $X$ is compact this action is subject to the usual axioms: $\alpha .(\beta . m)=(\alpha \beta) \cdot m$ and $1 . m=m$ for all 'scalars' $\alpha, \beta$ in $\mathcal{C}_{0}(X)$ and all elements $m$ of $M$. If $X$ is not compact, in which case the algebra $\mathcal{C}_{0}(X)$ does not have a unit element, the second axiom is replaced by the non-degeneracy requirement that $\left(\varphi_{j} . m\right)_{j}$ converges to $m$, where $\left(\varphi_{j}\right)_{j}$ is any contractive approximate identity for $\mathcal{C}_{0}(X)$. Clearly $\mathcal{C}_{0}(X)$ is a $\mathcal{C}_{0}(X)$-module under the pointwise operations.

Given two Banach modules $M$ and $N$ over $\mathcal{C}_{0}(X)$, a bounded linear map $T$ : $M \rightarrow N$ is said to be $\mathcal{C}_{0}(X)$-linear if $T(\alpha . m)=\alpha . T(m)$ for every scalar $\alpha \in \mathcal{C}_{0}(X)$ and every $m \in M$. The Banach space of all $\mathcal{C}_{0}(X)$-linear maps from $M$ to $N$ is denoted $L_{\mathcal{C}_{0}(X)}(M, N)$.

Received by the editors March 20, 2002.

2000 Mathematics Subject Classification. Primary 46B22; Secondary 46J10, 46E30.

Key words and phrases. Banach module, Radon-Nikodym Theorem, Riesz Theorem. 


\section{Generalized measures $\mu$ AND their $L^{1}(\mu)$-Spaces}

The starting point for our work is the fact that the continuous function $r: Y \rightarrow X$ yields a natural $\mathcal{C}_{0}(X)$-module structure on $\mathcal{C}_{0}(Y)$, the action being $\alpha \cdot f:=(\alpha \circ r) \cdot f$ for $\alpha \in \mathcal{C}_{0}(X)$ and $f \in \mathcal{C}_{0}(Y)$.

Consider the special case where $X$ is the one-point space $\{*\}$. Now the function $r: Y \rightarrow X$ is necessarily the constant function $r \equiv *$, the Banach algebra $\mathcal{C}_{0}(X)$ identifies with the field $\mathbb{C}$ and the action $\mathcal{C}_{0}(X) \times \mathcal{C}_{0}(Y) \rightarrow \mathcal{C}_{0}(Y)$ 'is' the vector space action of $\mathbb{C}$ on $\mathcal{C}_{0}(Y)$. Hence any $\mathcal{C}_{0}(X)$-linear map $\mu: \mathcal{C}_{0}(Y) \rightarrow \mathcal{C}_{0}(X)$ identifies with a usual bounded linear functional on $\mathcal{C}_{0}(Y)$. The Riesz Representation Theorem 4] in turn identifies $\mu$ with a finite Radon measure on $Y$.

This special case motivates

Definition 1.1. A generalized measure $\mu$ on $Y$ is a bounded $\mathcal{C}_{0}(X)$-linear map $\mu: \mathcal{C}_{0}(Y) \rightarrow \mathcal{C}_{0}(X)$. We say that $\mu$ is positive if $\mu(f) \geq 0$ for every $f \geq 0$.

Example 1.1. If $X$ is the one-point space, the generalized measures on $Y$ simply identify with the finite Radon measures on $Y$.

For more examples assume that $r: Y \rightarrow X$ is the canonical projection $\operatorname{pr}_{Z}: X \times Z$ $\rightarrow X$ for some locally compact space $Z$. Every Radon measure $\mu_{\mathrm{sc}}: \mathcal{C}_{0}(Z) \rightarrow \mathbb{C}$ on $Z$ induces a generalized measure $\mu: \mathcal{C}_{0}(X \times Z) \rightarrow \mathcal{C}_{0}(X)$ as the composite

$$
\mu: \mathcal{C}_{0}(X \times Z) \equiv \mathcal{C}_{0}(X) \check{\otimes} \mathcal{C}_{0}(Z) \stackrel{\operatorname{id}_{\mathcal{C}_{0}(X)} \check{\otimes} \mu_{\mathrm{sc}}}{\longrightarrow} \mathcal{C}_{0}(X) \check{\otimes} \mathbb{C} \equiv \mathcal{C}_{0}(X)
$$

We have

$$
\mu(f)(x)=\int_{Z} f(x, .) d \mu_{\mathrm{sc}}
$$

for $f \in \mathcal{C}_{0}(X \times Z)$ and $x \in X$. In particular $\mu$ is positive if $\mu_{\text {sc }}$ is positive.

To get more generalized measures on $X \times Z$ we can start with any bounded linear map $T_{\mu}: \mathcal{C}_{0}(Z) \rightarrow \mathcal{C}_{0}(X)$ : we obtain a generalized measure $\mu$ by taking the composite

$$
\mu: \mathcal{C}_{0}(X \times Z) \equiv \mathcal{C}_{0}(X) \check{\otimes} \mathcal{C}_{0}(Z) \stackrel{\operatorname{id}_{\mathcal{C}_{0}(X)} \ddot{\otimes} T_{\mu}}{\longrightarrow} \mathcal{C}_{0}(X) \check{\otimes} \mathcal{C}_{0}(X) \stackrel{\text { multiplication }}{\longrightarrow} \mathcal{C}_{0}(X) .
$$

We then have $\mu(f)(x)=T_{\mu}(f(x,)).(x)$ for $f \in \mathcal{C}_{0}(X \times Z)$ and $x \in X$. Again $\mu$ is positive if $T_{\mu}$ is positive.

It can be shown that every generalized measure $\mu: \mathcal{C}_{0}(X \times Z) \rightarrow \mathcal{C}_{0}(X)$ comes from a unique map $T_{\mu}: \mathcal{C}_{0}(Z) \rightarrow \mathcal{C}_{b}(X)$ : if $X$ is compact we simply have $T_{\mu}(g)=$ $\mu\left(g \circ \operatorname{pr}_{Z}\right)$ for $g \in \mathcal{C}_{0}(Z)$. If $X$ is merely locally compact, we use a contractive approximate identity of $\mathcal{C}_{0}(X)$ to obtain $T_{\mu}$. We have in fact an isometric identification of Banach lattices

$$
L_{\mathcal{C}_{0}(X)}\left(\mathcal{C}_{0}(X \times Z), \mathcal{C}_{0}(X)\right) \equiv L\left(\mathcal{C}_{0}(Z), \mathcal{C}_{b}(X)\right) .
$$

We now come to the definition of the space $L^{1}(\mu)$ for some fixed positive generalized measure $\mu$. To begin we observe that we can define a semi-norm on $\mathcal{C}_{0}(Y)$ by setting $\|f\|_{1}:=\|\mu(|f|)\|_{\mathcal{C}_{0}(X)}$, where $\|\cdot\|_{\mathcal{C}_{0}(X)}$ denotes the usual sup-norm. The subadditivity follows from the classical triangle inequality. Indeed, let $f, g$ be in $\mathcal{C}_{0}(Y)$. Since $\mu(|f+g|)$ is a positive element of $\mathcal{C}_{0}(X)$ there is some $x_{0}$ in $X$ such that $\|\mu(|f+g|)\|_{\mathcal{C}_{0}(X)}=\mu(|f+g|)\left(x_{0}\right)=\delta_{x_{0}} \circ \mu(|f+g|)$, where $\delta_{x_{0}}$ denotes the Dirac measure at $x_{0}$. As $\delta_{x_{0}} \circ \mu: \mathcal{C}_{0}(Y) \rightarrow \mathbb{C}$ 'is' a positive measure in the usual 
sense, the triangle inequality assures us that

$$
\begin{aligned}
\|f+g\|_{1} & =\delta_{x_{0}} \circ \mu(|f+g|)=\|f+g\|_{L^{1}\left(\delta_{x_{0}} \circ \mu\right)} \\
& \leq\|f\|_{L^{1}\left(\delta_{x_{0}} \circ \mu\right)}+\|g\|_{L^{1}\left(\delta_{x_{0}} \circ \mu\right)}=\delta_{x_{0}} \circ \mu(|f|)+\delta_{x_{0} \circ \mu} \circ(|g|) \\
& \leq\|\mu(|f|)\|_{\mathcal{C}_{0}(X)}+\|\mu(|g|)\|_{\mathcal{C}_{0}(X)} \\
& =\|f\|_{1}+\|g\|_{1} .
\end{aligned}
$$

We denote by $L^{1}(\mu)$ the completion of the normed space obtained from $\left(\mathcal{C}_{0}(Y)\right.$, $\left.\|.\|_{1}\right)$ by quotienting out the null-space of the semi-norm $\|\cdot\|_{1}$.

By the construction of our space $L^{1}(\mu)$ we have a canonical map $i: \mathcal{C}_{0}(Y) \rightarrow$ $L^{1}(\mu)$. This map is injective precisely when the semi-norm \|\|$_{1}$ on $\mathcal{C}_{0}(Y)$ is actually a norm, and this occurs when $\mu$ has "full support" in a sense which will be made precise in a subsequent paper [7].

Example 1.2. a) If $X$ is the one-point space our positive generalized measure $\mu$ identifies with a positive (finite) Radon measure on $Y$. The space $L^{1}(\mu)$ in the sense we just defined now identifies with the usual space $L^{1}(\mu)$.

b) It can be shown that in the case where $Y \stackrel{r}{\rightarrow} X$ is the identity $X \stackrel{\text { id }}{\rightarrow} X$, the space $L^{1}(\mu)$ identifies with a certain $\mathcal{C}_{0}(U)$. Specifically $U$ is the complement in $X$ of the subset $m^{-1}(\{0\})$, where $m \in \mathcal{C}_{b}(X)$ denotes the function such that $\mu(f)=f \cdot m$ for every $f \in \mathcal{C}_{0}(X)$.

Proposition 1.1. The space $L^{1}(\mu)$ is naturally a (non-degenerate) Banach $\mathcal{C}_{0}(X)$ module and a Banach lattice, in such a way that the canonical map $i: \mathcal{C}_{0}(Y) \rightarrow$ $L^{1}(\mu)$ is a morphism of $\mathcal{C}_{0}(X)$-modules and of Banach lattices.

Furthermore this map satisfies the universal property that for every Banach $\mathcal{C}_{0}(X)$-module $M$ and every contractive morphism $T:\left(\mathcal{C}_{0}(Y),\|.\|_{1}\right) \rightarrow M$ of seminormed $\mathcal{C}_{0}(X)$-modules there exists a unique contractive morphism $H: L^{1}(\mu) \rightarrow M$ of $\mathcal{C}_{0}(X)$-modules such that the following diagram commutes:

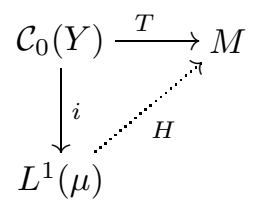

Proof. $\bullet L^{1}(\mu)$ is a $\mathcal{C}_{0}(X)$-module.

Recall that the $\mathcal{C}_{0}(X)$-module structure of the semi-normed space $\left(\mathcal{C}_{0}(Y),\|\cdot\|_{1}\right)$ is given by $\alpha \cdot f:=(\alpha \circ r) \cdot f$ for $\alpha \in \mathcal{C}_{0}(X)$ and $f \in \mathcal{C}_{0}(Y)$. As

$$
\begin{gathered}
\|\alpha . f\|_{1}=\|\mu(|\alpha \circ r \cdot f|)\|_{\mathcal{C}_{0}(X)}=\|\mu(|\alpha| \circ r \cdot|f|)\|_{\mathcal{C}_{0}(X)}=\|\mu(|\alpha| \cdot|f|)\|_{\mathcal{C}_{0}(X)} \\
=\||\alpha| \mu(|f|)\|_{\mathcal{C}_{0}(X)} \leq\|\alpha\|_{\mathcal{C}_{0}(X)}\|\mu(|f|)\|_{\mathcal{C}_{0}(X)}=\|\alpha\|_{\mathcal{C}_{0}(X)}\|f\|_{1},
\end{gathered}
$$

the nullspace $K:=\|\cdot\|_{1}^{-1}(\{0\})$ of our semi-norm $\|\cdot\|_{1}$ is algebraically an ideal in $\mathcal{C}_{0}(Y)$, so the module action of $\mathcal{C}_{0}(X)$ on $\mathcal{C}_{0}(Y)$ quotients to a $\mathcal{C}_{0}(X)$-action on $\mathcal{C}_{0}(Y) / K$. Furthermore we still have $\|\alpha . f\| \leq\|\alpha\|_{\mathcal{C}_{0}(X)}\|f\|$ in $\mathcal{C}_{0}(Y) / K$. It follows that the completion $L^{1}(\mu)$ of this quotient space is still a $\mathcal{C}_{0}(X)$-module (with contractive action). The map $i: \mathcal{C}_{0}(Y) \rightarrow L^{1}(\mu)$ is obviously a morphism of $\mathcal{C}_{0}(X)$ modules and satisfies the asserted property.

We now show that $L^{1}(\mu)$ is in fact a non-degenerate $\mathcal{C}_{0}(X)$-module in the sense that $\left(\varphi_{j} . f\right)_{j}$ converges to $f$ for every $f \in L^{1}(\mu)$, where $\left(\varphi_{j}\right)_{j}$ is any contractive approximate identity of $\mathcal{C}_{0}(X)$. As the bounded canonical map $i:\left(\mathcal{C}_{0}(Y),\|\cdot\|_{\mathcal{C}_{0}(Y)}\right) \rightarrow$ 
$\left(L^{1}(\mu),\|\cdot\|_{1}\right)$ has dense range it suffices to consider elements $f=i(g)$ for some compactly supported $g$ in $\mathcal{C}_{0}(Y)$. By taking an appropriate contractive approximate identity we can assume that for all $j$ greater than or equal to some $j_{0}$ the function $\varphi_{j}$ is identically equal to 1 on $r(\operatorname{supp}(g))$. We then have $\varphi_{j} . i(g)=i(g)$, so $\varphi_{j} . i(g) \underset{j}{\rightarrow} i(g)$.

- $L^{1}(\mu)$ is a Banach lattice.

The nullspace $K:=\|\cdot\|_{1}^{-1}(\{0\})$ of the semi-norm $\|.\|_{1}$ is a lattice ideal in $\left(\mathcal{C}_{0}(Y), \leq\right)$, since if $0 \leq f \leq g$ and $g$ belongs to $K$, then $f$ also belongs to $K$. Therefore the quotient $\mathcal{C}_{0}(Y) / K$ is naturally a normed lattice, and so is its completion $L^{1}(\mu)$. The map $i$ is obviously a morphism of lattices.

Remark. For future reference (Definition 2.2) we observe that $L^{1}(\mu)$ is even a $\mathcal{C}_{0}(Y)$ module in the natural way: given $\alpha \in \mathcal{C}_{0}(Y)$ and $g \in L^{1}(\mu)$ of the form $i(f)$ for some $f \in \mathcal{C}_{0}(Y)$, we have $\alpha . g=i(\alpha \cdot f)$. Using the projective tensor product to linearize the action, we get a contraction $\mathcal{C}_{0}(Y) \hat{\otimes} L^{1}(\mu) \rightarrow L^{1}(\mu)$ which is $\mathcal{C}_{0}(X)$ linear when we consider on $\mathcal{C}_{0}(Y) \hat{\otimes} L^{1}(\mu)$ the $\mathcal{C}_{0}(X)$-module structure it inherits from $\mathcal{C}_{0}(Y)$.

We can now state the following

Proposition 1.2. The positive generalized measure $\mu: \mathcal{C}_{0}(Y) \rightarrow \mathcal{C}_{0}(X)$ induces a unique positive, contractive, $\mathcal{C}_{0}(X)$-linear map $\tilde{\mu}: L^{1}(\mu) \rightarrow \mathcal{C}_{0}(X)$ such that the diagram

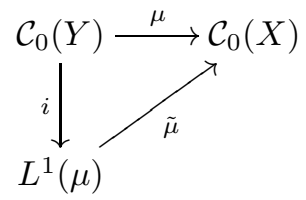

commutes.

Furthermore we have $\|f\|_{L^{1}(\mu)}=\|\tilde{\mu}(|f|)\|_{\mathcal{C}_{0}(X)}$ for every $f$ in $L^{1}(\mu)$, where $|f|$ denotes the absolute value of $f$ for the lattice structure of $L^{1}(\mu)$.

We stress that $\tilde{\mu}$ is contractive, whatever the norm of $\mu$. This is possible because $\mu$ already intervenes when we compute the norm of an element of $L^{1}(\mu)$.

In practice we might use the letter $\mu$ to denote both the positive generalized measure $\mu$ and the map $\tilde{\mu}$ of the proposition.

Proof. - To prove the first part of the proposition it is enough to show that $\mu$ is contractive when we consider it as a map $\left(\mathcal{C}_{0}(Y),\|\cdot\|_{1}\right) \rightarrow\left(\mathcal{C}_{0}(X),\|\cdot\|_{\mathcal{C}_{0}(X)}\right)$ between semi-normed spaces. Let $f \in \mathcal{C}_{0}(Y)$. We need to show that $\|\mu(f)\|_{\mathcal{C}_{0}(X)} \leq$ $\|\mu(|f|)\|_{\mathcal{C}_{0}(X)}$. We choose $x_{0}$ in $X$ and $c \in \mathbb{C}$ of modulus one such that $\mu(c . f)\left(x_{0}\right)=$ $\|\mu(f)\|_{\mathcal{C}_{0}(X)}$.

Since $\delta_{x_{0}} \circ \mu: \mathcal{C}_{0}(Y) \rightarrow \mathbb{C}$ is a positive Radon measure we have

$$
\begin{aligned}
\|\mu(f)\|_{\mathcal{C}_{0}(X)} & =\mu(c . f)\left(x_{0}\right)=\delta_{x_{0}} \circ \mu(c . f)=\int_{Y} c . f d\left(\delta_{x_{0}} \circ \mu\right) \leq \int_{Y}|c . f| d\left(\delta_{x_{0}} \circ \mu\right) \\
& =\delta_{x_{0}}(\mu(|c . f|)) \leq\|\mu(|c . f|)\|_{\mathcal{C}_{0}(X)}=\|\mu(|f|)\|_{\mathcal{C}_{0}(X)} .
\end{aligned}
$$

- Let us prove that $\|f\|_{L^{1}(\mu)}=\|\tilde{\mu}(|f|)\|_{\mathcal{C}_{0}(X)}$ for every $f \in L^{1}(\mu)$. As the isometry $i:\left(\mathcal{C}_{0}(Y),\|\cdot\|_{1}\right) \rightarrow L^{1}(\mu)$ has dense range and since $f \mapsto|f|$ is continuous 
it is sufficient to verify the assertion for $f=i(g), g \in \mathcal{C}_{0}(Y)$. But in this case

$$
\begin{gathered}
\|\tilde{\mu}(|f|)\|_{\mathcal{C}_{0}(X)}=\|\tilde{\mu}(|i(g)|)\|_{\mathcal{C}_{0}(X)}=\|\tilde{\mu}(i(|g|))\|_{\mathcal{C}_{0}(X)} \\
=\|\mu(|g|)\|_{\mathcal{C}_{0}(X)}=\|g\|_{1}=\|i(g)\|_{L^{1}(\mu)}=\|f\|_{L^{1}(\mu)} .
\end{gathered}
$$

We should remark that if $\mu: \mathcal{C}_{0}(Y) \rightarrow \mathcal{C}_{0}(X)$ is merely a positive bounded map the construction of the Banach lattice $L^{1}(\mu)$ still makes sense and we still get a contractive positive map $\tilde{\mu}: L^{1}(\mu) \rightarrow \mathcal{C}_{0}(X)$ as in the proposition.

We have already seen what our space $L^{1}(\mu)$ looks like in two special cases. The following proposition shows that these are actually extreme cases, with $X$ and $Z$ respectively being the one-point space.

Proposition 1.3. Suppose $r: Y \rightarrow X$ is the canonical projection $X \times Z \rightarrow X$ with $Z$ being some locally compact space. Additionally assume that the positive generalized measure $\mu: \mathcal{C}_{0}(X \times Z) \rightarrow \mathcal{C}_{0}(X)$ arises from some positive Radon measure $\mu_{s c}: \mathcal{C}_{0}(Z) \rightarrow \mathbb{C}$. Then we have the natural isometric $\mathcal{C}_{0}(X)$-module and lattice identification $L^{1}(\mu) \equiv \mathcal{C}_{0}\left(X, L^{1}\left(Z, \mu_{s c}\right)\right)$.

By $L^{1}\left(Z, \mu_{\mathrm{sc}}\right)$ we mean the usual space $L^{1}$ coming from the measure $\mu_{\mathrm{sc}}$ on $Z$.

The way $\mu$ arises from $\mu_{\mathrm{sc}}$ was illustrated when we saw examples of generalized measures. Recall in particular that $\mu(f)(x)=\int_{Z} f(x,.) d \mu_{\mathrm{sc}}$.

Proof. Let $j: \mathcal{C}_{0}(Z) \rightarrow L^{1}\left(Z, \mu_{\mathrm{sc}}\right)$ be the canonical map. We define a morphism of $\mathcal{C}_{0}(X)$-modules $T: \mathcal{C}_{0}(X \times Z) \rightarrow \mathcal{C}_{0}\left(X, L^{1}\left(Z, \mu_{\mathrm{sc}}\right)\right)$ as the composite

$$
T: \mathcal{C}_{0}(X \times Z) \equiv \mathcal{C}_{0}\left(X, \mathcal{C}_{0}(Z)\right) \stackrel{\mathcal{C}_{0}(X, j)}{\longrightarrow} \mathcal{C}_{0}\left(X, L^{1}\left(Z, \mu_{\mathrm{sc}}\right)\right),
$$

where $\mathcal{C}_{0}(X, j)$ denotes the map $g \mapsto j \circ g$.

This morphism $T$ is isometric as a map $\left(\mathcal{C}_{0}(X \times Z),\|\cdot\|_{1}\right) \rightarrow \mathcal{C}_{0}\left(X, L^{1}\left(Z, \mu_{\mathrm{sc}}\right)\right)$.

Indeed, fix $f \in \mathcal{C}_{0}(X \times Z)$ and $x \in X$ : we have $T(f)(x)=j(f(x,)$.$) in L^{1}\left(Z, \mu_{\mathrm{sc}}\right)$. Hence

$$
\begin{aligned}
\|T(f)(x)\|_{L^{1}\left(Z, \mu_{\mathrm{sc}}\right)} & =\int_{Z}|T(f)(x)| d \mu_{\mathrm{sc}}=\int_{Z}|j(f(x, .))| d \mu_{\mathrm{sc}} \\
& =\int_{Z}|f|(x, .) d \mu_{\mathrm{sc}}=\mu(|f|)(x) .
\end{aligned}
$$

Therefore $\|T(f)\|=\|\mu(|f|)\|_{\mathcal{C}_{0}(X)}=\|f\|_{1}$ and $T$ is isometric.

As $T$ is a contractive morphism of $\mathcal{C}_{0}(X)$-modules there is a unique contractive morphism of $\mathcal{C}_{0}(X)$-modules $H: L^{1}(\mu) \rightarrow \mathcal{C}_{0}\left(X, L^{1}\left(Z, \mu_{\mathrm{sc}}\right)\right)$ such that the following diagram commutes:

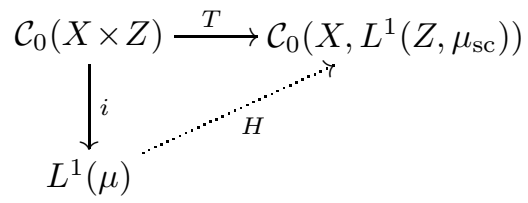

Since $T$ is an isometry and the isometry $i$ has dense range it follows that $H$ is also an isometry. $H$ is clearly a morphism of lattices.

It remains to show that $H$ is onto, and for this it is sufficient to show that $T$ has dense range. But this follows from the facts that $j: \mathcal{C}_{0}(Z) \rightarrow L^{1}\left(Z, \mu_{\mathrm{sc}}\right)$ has dense range and that the map $T$ can also be described as

$$
T: \mathcal{C}_{0}(X \times Z) \equiv \mathcal{C}_{0}(X) \check{\otimes} \mathcal{C}_{0}(Z) \stackrel{\operatorname{id}_{\mathcal{C}_{0}(X)} \check{\otimes} j}{\longrightarrow} \mathcal{C}_{0}(X) \check{\otimes} L^{1}\left(Z, \mu_{\mathrm{sc}}\right) \equiv \mathcal{C}_{0}\left(X, L^{1}\left(Z, \mu_{\mathrm{sc}}\right)\right) .
$$




\section{Absolute Continuity And Densities}

We continue to work with some fixed continuous map $r: Y \rightarrow X$ between locally compact spaces. Also $\mu$ and $\nu$ denote two positive generalized measures $\mathcal{C}_{0}(Y) \rightarrow \mathcal{C}_{0}(X)$.

As we cannot use Borel sets to define what it would mean for $\nu$ to be absolutely continuous with respect to $\mu$, we adopt the following

Definition 2.1. We say that $\nu$ is absolutely continuous with respect to $\mu$ if for every $\epsilon>0$ there is an $\eta>0$ such that for every continuous function $f$ in $\mathcal{C}_{0}(Y)$ with $0 \leq f \leq 1,\|\mu(f)\|_{\mathcal{C}_{0}(X)}<\eta$ implies $\|\nu(f)\|_{\mathcal{C}_{0}(X)}<\epsilon$.

This definition is justified by

Proposition 2.1. Suppose that $X$ is the one-point space, so that the positive generalized measures $\mu, \nu: \mathcal{C}_{0}(Y) \rightarrow \mathcal{C}_{0}(X) \equiv \mathbb{C}$ identify with usual positive Radon measures on $Y$. The generalized measure $\nu$ is absolutely continuous with respect to the generalized measure $\mu$ in the sense of the above definition if and only if the Radon measure $\nu$ is absolutely continuous with respect to the Radon measure $\mu$ in the usual sense.

Proof. • Absolute continuity in the traditional sense implies absolute continuity in the above sense.

We can assume that $\nu(Y)>0$. Let $\epsilon>0$ be given. There exists a $\tilde{\eta}>0$ such that for every Borel set $A \subseteq Y, \mu(A) \leq \tilde{\eta}$ implies $\nu(A)<\epsilon / 2$. We show that $\eta:=\epsilon \tilde{\eta} / 2 \nu(Y)$ works in the above definition.

Let $f \in \mathcal{C}_{0}(Y), 0 \leq f \leq 1$, with $\mu(f)<\eta$. We then have

$$
\mu\left(\left[f \geq \frac{\epsilon}{2 \nu(Y)}\right]\right)=\int_{[f \geq \epsilon / 2 \nu(Y)]} d \mu \leq \frac{2 \nu(Y)}{\epsilon} \int_{[f \geq \epsilon / 2 \nu(Y)]} \underset{f}{f} d \mu \leq \frac{2 \nu(Y)}{\epsilon} \eta=\tilde{\eta} .
$$

Hence $\nu\left(\left[f \geq \frac{\epsilon}{2 \nu(Y)}\right]\right)<\frac{\epsilon}{2}$, and so

$$
\begin{aligned}
\nu(f) & =\int_{[1 \geq f \geq \epsilon / 2 \nu(Y)]} \underset{[f<\epsilon / 2 \nu(Y)]}{f} d \nu \\
& \leq \nu\left(\left[f \geq \frac{\epsilon}{2 \nu(Y)}\right]\right)+\int_{Y} \frac{\epsilon}{2 \nu(Y)} d \nu<\frac{\epsilon}{2}+\frac{\epsilon}{2 \nu(Y)} \nu(Y)=\epsilon .
\end{aligned}
$$

- Absolute continuity in the above sense implies absolute continuity in the traditional sense.

Fix $\epsilon>0$. Since $\nu$ and $\mu$ are inner regular it is enough to show that there exists an $\eta>0$ such that $\mu(K)<\eta$ implies $\nu(K)<\epsilon$ for all compact subsets $K$ of $Y$.

There is $\tilde{\eta}>0$ such that $\mu(f)<\tilde{\eta}$ implies $\nu(f)<\epsilon$ for every $f$ in $\mathcal{C}_{0}(Y)$, $0 \leq f \leq 1$. Then $\eta:=\tilde{\eta} / 2$ works. To see this let $K \subseteq Y$ be compact, with $\mu(K)<\eta$. As $\mu$ is outer regular there exists an open subset $U$ of $Y$ containing $K$ such that $\mu(U)<\tilde{\eta}$. Next, there is a continuous function $f$ on $Y, 0 \leq f \leq 1$, such that $f \equiv 1$ on $K$ and $f \equiv 0$ on $X \backslash U$.

Since $f \leq 1_{U}$ it follows that $\mu(f) \leq \mu(U)<\tilde{\eta}$, and hence $\nu(f)<\epsilon$. But $1_{K} \leq f$, so $\nu(K) \leq \nu(f)<\epsilon$.

Remark. The definition of absolute continuity for generalized measures has been chosen in a way such that the proposition holds. One might be inclined to define $\nu$ as absolutely continuous with respect to $\mu$ if and only if for every continuous function 
$f$ on $Y$, with $0 \leq f \leq 1, \mu(f)=0$ implies $\nu(f)=0$. To see that this is not a good definition take for $X$ the one-point space and for $Y$ the real line. Let $\left(q_{n}\right)_{n}$ be an enumeration of the rational numbers. Then the Lebesgue measure and the measure $\sum_{n} \frac{1}{2^{n}} \delta_{q_{n}}$ would be mutually absolutely continuous (the only continuous positive function they annihilate is the zero function), although neither one is absolutely continuous with respect to the other in the usual sense.

We now generalize the concept of density in our setting. Recall from the Remark before Proposition 1.2 that $L^{1}(\mu)$ is a $\mathcal{C}_{0}(Y)$-module and that the contractive actionmap $\mathcal{C}_{0}(Y) \hat{\otimes} L^{1}(\mu) \rightarrow L^{1}(\mu)$ is $\mathcal{C}_{0}(X)$-linear. Also recall the $\mathcal{C}_{0}(X)$-linear contraction $\tilde{\mu}: L^{1}(\mu) \rightarrow \mathcal{C}_{0}(X)$ of Proposition 1.2. Any $d \in L^{1}(\mu)$ yields a composite morphism of $\mathcal{C}_{0}(X)$-modules

$$
\mathcal{C}_{0}(Y) \stackrel{f \mapsto f \otimes d}{\longrightarrow} \mathcal{C}_{0}(Y) \hat{\otimes} L^{1}(\mu) \stackrel{\text { action }}{\longrightarrow} L^{1}(\mu) \stackrel{\tilde{\mu}}{\longrightarrow} \mathcal{C}_{0}(X)
$$

which is the subject of the following

Definition 2.2. Given $d$ in $L^{1}(\mu)$, the (not necessarily positive) generalized measure $d . \mu: \mathcal{C}_{0}(Y) \rightarrow \mathcal{C}_{0}(X)$ with density $d$ with respect to $\mu$ is defined as the above composite map. Hence $d . \mu$ is given by

$$
d \cdot \mu(f)=\tilde{\mu}(f \cdot d) .
$$

Clearly $d . \mu$ has norm at most $\|d\|_{L^{1}(\mu)}$. The following proposition asserts that equality holds. In analogy with the classical case let us denote the "dual" of $\mathcal{C}_{0}(Y)$, the space $L_{\mathcal{C}_{0}(X)}\left(\mathcal{C}_{0}(X \times Z), \mathcal{C}_{0}(X)\right)$ of all $\mathcal{C}_{0}(X)$-linear maps $\mathcal{C}_{0}(Y) \rightarrow \mathcal{C}_{0}(X)$, by $\mathcal{C}_{0}(Y)^{*}$. Since the algebra $\mathcal{C}_{0}(X)$ is commutative, $\mathcal{C}_{0}(Y)^{*}$ is still a $\mathcal{C}_{0}(X)$-module. As in the classical situation we have

Proposition 2.2. The map $d \mapsto d . \mu$ is an isometric embedding of Banach lattices and $\mathcal{C}_{0}(X)$-modules

$$
L^{1}(\mu) \hookrightarrow \mathcal{C}_{0}(Y)^{*} .
$$

Proof. • The map is isometric.

As we noted before, it is contractive. We can assume that $\mu \neq 0$. Let $d$ in $L^{1}(\mu)$ and fix $\epsilon>0$. We need to show that $\|d . \mu\| \geq\|d\|_{L^{1}(\mu)}-\epsilon$. Now, since the isometry $i:\left(\mathcal{C}_{0}(Y),\|\cdot\|_{1}\right) \rightarrow L^{1}(\mu)$ has dense range, there exists some $\tilde{d}$ in $\mathcal{C}_{0}(Y)$ such that $\|d-i(\tilde{d})\|_{L^{1}(\mu)}<\epsilon / 3$. For simplicity we will write $\tilde{d}$ instead of $i(\tilde{d})$. Next, as the subset $[|\tilde{d}| \geq \epsilon / 6\|\mu\|]$ of $Y$ is compact, there exists some $\varphi$ in $\mathcal{C}_{0}(Y), 0 \leq|\varphi| \leq 1$, such that $(\varphi \cdot \tilde{d})(y)=|\tilde{d}|(y)$ for every $y$ for which $|d|(y) \geq \epsilon / 6\|\mu\|$. Therefore we have that $\|\varphi \cdot \tilde{d}-|\tilde{d}|\|_{\mathcal{C}_{0}(Y)} \leq \epsilon / 3\|\mu\|$. It follows that

$$
\begin{aligned}
\|d . \mu\| & \geq\|d . \mu(\varphi)\|=\|\tilde{\mu}(\varphi \cdot d)\| \\
& \geq\|\tilde{\mu}(|\tilde{d}|)\|-\|\mu(\varphi \cdot \tilde{d}-|\tilde{d}|)\|-\|\tilde{\mu}(\varphi \cdot(d-\tilde{d}))\| \\
& \geq\|\tilde{d}\|_{L^{1}(\mu)}-\|\mu\| \cdot\|\varphi \cdot \tilde{d}-|\tilde{d}|\|_{\text {sup }}-\|\tilde{\mu}\| \cdot\|\varphi\|_{\text {sup }} \cdot\|d-\tilde{d}\|_{L^{1}(\mu)} \\
& \geq\|\tilde{d}\|_{L^{1}(\mu)}-\|\mu\| \cdot \frac{\epsilon}{3\|\mu\|}-1 \cdot 1 \cdot \frac{\epsilon}{3} \geq\left(\|d\|_{L^{1}(\mu)}-\frac{\epsilon}{3}\right)-\frac{\epsilon}{3}-\frac{\epsilon}{3} \\
& =\|d\|_{L^{1}(\mu)}-\epsilon .
\end{aligned}
$$

- The map is an embedding of lattices.

It is obvious from the definition of the lattice structure of $L^{1}(\mu)$ that a positive $d$ in $L^{1}(\mu)$ yields a positive generalized measure $d . \mu$. Now fix $d \in L^{1}(\mu)$ and suppose 
that $d . \mu$ is positive. We need to show that $d$ is positive in $L^{1}(\mu)$. For this we show that $d=|d|$ in $L^{1}(\mu)$.

When the product space $\prod_{x \in X} L^{1}\left(\delta_{x} \circ \mu\right)$ is endowed with the sup-norm the natural embedding $L^{1}(\mu) \hookrightarrow \prod_{x \in X} L^{1}\left(\delta_{x} \circ \mu\right)$ is injective. Hence it is enough to show that $d$ equals $|d|$ in $L^{1}\left(\delta_{x} \circ \mu\right)$ for fixed $x \in X$. But this follows from the fact that the measure $\delta_{x} \circ(d . \mu)$ is positive on $\mathcal{C}_{0}(Y)$ : since $\int_{Y} f d d\left(\delta_{x} \circ \mu\right) \geq 0$ for every positive $f$ in $\mathcal{C}_{0}(Y), d$ is positive $\delta_{x} \circ \mu$-a.e.

As we might expect we still have the following

Proposition 2.3. If a positive generalized measure $\nu=d$. $\mu$ has a density $d \in L^{1}(\mu)$ with respect to $\mu$, then $\nu$ is absolutely continuous with respect to $\mu$.

Proof. Let $\epsilon>0$ be fixed. Since $\nu$ is positive we know from Proposition 2.2 that $d$ is also positive. Hence there is a positive $\tilde{d}$ in $\mathcal{C}_{0}(Y)$ such that $\|d-\tilde{d}\|_{L^{1}(\mu)}<\epsilon / 2$. Take $\eta>0$ such that $\|\tilde{d}\|_{\mathcal{C}_{0}(Y)} \cdot \eta<\epsilon / 2$. Then, for every $f$ in $\mathcal{C}_{0}(Y), 0 \leq f \leq 1$, with $\|\mu(f)\|<\eta$ we have

$$
\begin{aligned}
\|\nu(f)\| & =\|\tilde{\mu}(f \cdot d)\| \leq\|\tilde{\mu}(f \cdot(d-\tilde{d}))\|+\|\mu(f \cdot \tilde{d})\| \\
& \leq \frac{\epsilon}{2}+\|\tilde{d}\|_{\mathcal{C}_{0}(Y)}\|\mu(f)\|_{\mathcal{C}_{0}(X)} \leq \frac{\epsilon}{2}+\|\tilde{d}\| \cdot \eta \\
& <\frac{\epsilon}{2}+\frac{\epsilon}{2}=\epsilon .
\end{aligned}
$$

\section{RADON-NIKODYM FAILS}

We now arrive at the main result of this paper. In the classical situation, which corresponds to the case where $X$ is the one-point space, the Radon-Nikodym Theorem [5] asserts the converse to Proposition 2.3: if $\nu$ is absolutely continuous with respect to $\mu$, then it has a density in $L^{1}(\mu)$. The following theorem shows that this converse is no longer true in our setting.

Theorem 3.1. There are compact spaces $X, Z$ and positive generalized measures $\mu, \nu: \mathcal{C}_{0}(X \times Z) \rightarrow \mathcal{C}_{0}(X)$ such that $\nu$ is absolutely continuous with respect to $\mu$, but has no density $d \in L^{1}(\mu)$.

In order to give such a counter-example we need the following

Lemma 3.1. There exists a family $\left(g_{n}\right)_{n \in \mathbb{N} \cup\{\infty\}}$ of positive functions in $L^{1}(0,1)$, taking values $\leq 1$, such that $\left(g_{n}\right)_{n \in \mathbb{N}}$ converges to $g_{\infty}$ in the weak-star topology, but not in norm. By the weak-star topology we mean the subspace topology on $L^{1}(0,1)$ induced by the weak star-topology on $\mathcal{C}_{0}([0,1])^{*}$.

Proof of Lemma 3.1. The functions $g_{n}(t):=\frac{1}{2}(1+\cos (2 n \pi t))$ for $n \in \mathbb{N}$ and $g_{\infty}(t):=\frac{1}{2}$ satisfy the conditions: they are clearly positive and take values $\leq 1$. Since $t \mapsto \cos (2 \pi n t)$ is the real part of $t \mapsto e^{i 2 \pi n t}$ it follows from the RiemannLebesgue lemma that the sequence $(t \mapsto \cos (2 \pi n t))_{n}$ converges to 0 in the weakstar topology. Hence $\left(g_{n}\right)_{n \in \mathbb{N}}$ converges weak-star to $g_{\infty}$. But it is easy to compute that $\left\|g_{n}-g_{\infty}\right\|_{L^{1}}=1 / \pi$ for all $n \in \mathbb{N}$, so that there is no convergence in norm.

Proof of Theorem 3.1. To prove the theorem we consider the following:

- We take for $X$ the one-point-compactification $X:=\mathbb{N} \cup\{\infty\}$ of the integers. Hence the space $\mathcal{C}_{0}(X)$ can be identified with the space $c$ of all convergent sequences.

- We choose $Z$ to be the closed interval $[0,1]$. 
- We now define $\nu: \mathcal{C}_{0}(X \times Z) \rightarrow \mathcal{C}_{0}(X)$ by setting

$$
\nu(f)(n):=\int_{[0,1]} f(n, .) g_{n} d \lambda
$$

for $f \in \mathcal{C}_{0}(X \times Z)$ and $n \in X$, where $\lambda$ denotes Lebesgue measure. This map $\nu$ is well-defined: as $\infty$ is the only accumulation point of $X$, to verify that $\nu(f)$ is continuous on $X$ it is enough to show that $(\nu(f)(n))_{n \in \mathbb{N}}$ converges to $\nu(f)(\infty)$. But, as $f$ belongs to $\mathcal{C}_{0}(X \times Z) \equiv \mathcal{C}_{0}\left(X, \mathcal{C}_{0}(Z)\right)$, for given $\epsilon>0$ there is an $n_{1} \in \mathbb{N}$ such that $\|f(n, .)-f(\infty, .)\|_{\mathcal{C}_{0}(Z)}<\epsilon / 2$ for all $n \geq n_{1}$.

Next, as $\left(g_{n}\right)_{n}$ converges weak-star to $g_{\infty}$, there is $n_{2} \in \mathbb{N}$ such that

$$
\left|\int_{[0,1]} f(\infty, .)\left(g_{n}-g_{\infty}\right) d \lambda\right|<\epsilon / 2
$$

for all $n \geq n_{2}$. Hence for all $n \geq N:=\max \left\{n_{1}, n_{2}\right\}$, we have

$$
\begin{aligned}
& |\nu(f)(n)-\nu(f)(\infty)|=\left|\int_{[0,1]} f(n, .) g_{n} d \lambda-\int_{[0,1]} f(\infty, .) g_{\infty} d \lambda\right| \\
\leq & \left|\int_{[0,1]}[f(n, .)-f(\infty, .)] g_{n} d \lambda\right|+\left|\int_{[0,1]} f(\infty, .)\left[g_{n}-g_{\infty}\right] d \lambda\right| \leq \frac{\epsilon}{2}+\frac{\epsilon}{2}=\epsilon .
\end{aligned}
$$

It is now obvious that $\nu$ is bounded (in fact contractive), positive and $\mathcal{C}_{0}(X)$ linear, so $\nu$ is a positive generalized measure.

- For the positive generalized measure $\mu$ we simply take the one which comes from $\mu_{\mathrm{sc}}:=\lambda$, the Lebesgue measure on $Z:=[0,1]$.

Hence we have $\mu(f)(n)=\int_{[0,1]} f(n,). d \lambda$ for $f \in \mathcal{C}_{0}(X \times Z)$ and $n \in X$.

Having chosen the spaces $X, Z$ and the positive generalized measures $\nu$ and $\mu$ we now show that $\nu$ is absolutely continuous with respect to $\mu$, but does not have a density.

- $\nu$ is absolutely continuous with respect to $\mu$.

Given $\epsilon>0$, we can take $\eta:=\epsilon$ to ensure that for every $f \in \mathcal{C}_{0}(X \times Z), 0 \leq f \leq$ $1,\|\mu(f)\|<\eta$ implies $\|\nu(f)\|<\epsilon$. Indeed, evaluating $\nu(f)$ at any $n \in X$, we have

$$
0 \leq \nu(f)(n)=\int_{[0,1]} f(n, .) g_{n} d \lambda \leq \int_{[0,1]} f(n, .) d \lambda=\mu(f)(n) \leq\|\mu(f)\|<\eta=\epsilon .
$$

- $\nu$ does not have a density $d \in L^{1}(\mu)$ with respect to $\mu$.

By Proposition 1.3 we know that $L^{1}(X \times Z, \mu)$ identifies with $\mathcal{C}_{0}\left(X, L^{1}(0,1)\right)$. Arguing by contradiction, we assume that $\nu$ does have a density $d \in \mathcal{C}_{0}\left(X, L^{1}(0,1)\right)$ with respect to $\mu$.

For every $n \in X$ and every $g \in \mathcal{C}_{0}(Z)$ we then have, using the canonical projection $\operatorname{pr}_{Z}: X \times Z \rightarrow Z$,

$$
\begin{gathered}
\int_{[0,1]} g \cdot d(n) d \lambda=\int_{[0,1]} g \circ \operatorname{pr}_{Z}(n, .) \cdot d(n) d \lambda=\tilde{\mu}\left(g \circ \mathrm{pr}_{Z} \cdot d\right)(n) \\
\quad=\nu\left(g \circ \operatorname{pr}_{Z}\right)(n)=\int_{[0,1]} g \circ \operatorname{pr}_{Z}(n, .) \cdot g_{n} d \lambda=\int_{[0,1]} g \cdot g_{n} d \lambda .
\end{gathered}
$$

Hence $d(n)=g_{n} \lambda$-a.e., for all $n \in X$. As $d: X \rightarrow L^{1}(0,1)$ is continuous, the sequence $(d(n))_{n}$ converges in norm to $d(\infty)$. But this contradicts the fact that $\left(g_{n}\right)_{n \in \mathbb{N}}$ does not converge in norm. 


\section{ACKNOWLEDGMENT}

The author thanks his advisor, Andrew M. Tonge, for providing encouragement as well as numerous helpful suggestions and improvements.

\section{REFERENCES}

[1] David P. Blecher, Paul S. Muhly, Vern I. Paulsen, Categories of Operator Modules (Morita Equivalence and Projective Modules), Memoirs of the American Mathematical Society 143 (2000). MR 2000j:46132

[2] Johann Cigler, Viktor Losert, Peter Michor, Banach Modules and Functors on Categories of Banach Spaces, Lecture Notes in Pure and Applied Mathematics 46 (1979). MR 80j:46112

[3] Joseph Diestel, John J. Uhl, Vector Measures, American Mathematical Society, Mathematical Surveys 15 (1977). MR 56:2216

[4] Gerald B. Folland, Real Analysis, John Wiley \& Sons, Inc. (1999). MR 2000c:00001

[5] Paul Malliavin, Integration and Probability, Graduate Texts in Mathematics 157, Springer Verlag (1995). MR 97f:28001a

[6] Helmut H. Schaefer, Banach Lattices and Positive Operators, Springer Verlag (1974). MR 54:11023

[7] Gerd Zeibig, Generalized $L^{p}(\mu)$-spaces, to appear.

Department of Mathematical Sciences, Kent State University, Kent, Ohio 44240

E-mail address: gzeibig@math.kent.edu 